\title{
Innovative cold joining technologies based on tube forming
}

\author{
L.M. Alves ${ }^{\mathrm{a}}$, C.M.A. Silva, and P.A.F. Martins \\ IDMEC, Instituto Superior Técnico, Universidade de Lisboa, Av. Rovisco Pais, 1049-001 Lisboa, \\ Portugal
}

\begin{abstract}
This paper is focused on innovative cold joining technologies for connecting tubes and fixing tubes to sheets. The proposed technologies are based on the utilization of plastic instability waves in thin-walled tubes subjected to axial compression and may be seen as an alternative to conventional joining technologies based on mechanical fixing with fasteners, welding and structural adhesive bonding. Besides allowing connecting dissimilar materials and being successfully employed in fixture conditions that are difficult and costly to achieve by means of conventional joining the new proposed technologies also cope with the growing concerns on the demand, lifecycle and recycling of materials.
\end{abstract}

\section{Introduction}

Conventional technologies for connecting tubes are widely utilized in plumbing, air conditioning, refrigeration, process piping and lightweight structures, among other applications. The most wellknown technologies are based on commercially available tee fittings, saddle adapters and weld-o-lets for standard geometries and materials, such as carbon steel, stainless steel and copper (Figs. 1a to 1c). A standard tee fitting (Fig. 1a) has three welds; two in the main tube and one in the branch tube. Saddle adapters or weld-o-lets (Figs. 1b and 1c) also need to be brazed or welded to the main tube over a pre-cut hole and the attachment to the branch tube is made through a weld or a threated connection.

Custom based solutions are mainly based on nozzle-weld and spin-forming (Figs. 1d and 1e). Nozzle-weld connections (Fig. 1d) require cutting a hole in the main tube, shaping a contoured end in the branch tube to match the diameter of the main tube and welding along the contour. Spin-forming (Fig. 1e) also requires cutting a hole in the main tube but the difference is that material around that hole is subsequently shaped into a tee fitting where the branch tube will be brazed or welded.

In case of fixing tubes to sheets, the most widespread technologies are based on the utilization of mechanical fixing with fasteners (nuts and bolts or rivets), welding, and structural adhesive bonding (Figs. 1g to 1i).

No matter the application and the differences between the technologies that are currently available for connecting tubes (Figs. 1a to 1e) and fixing tubes to sheets (Figs. 1g to 1i), their universe of applicability is always limited by aesthetic, physical, chemical and mechanical requirements.

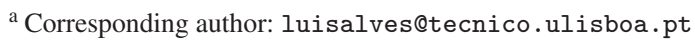

This is an Open Access article distributed under the terms of the Creative Commons Attribution License 4.0, which permits unrestricted use, distribution, and reproduction in any medium, provided the original work is properly cited. 


\section{MATEC Web of Conferences}
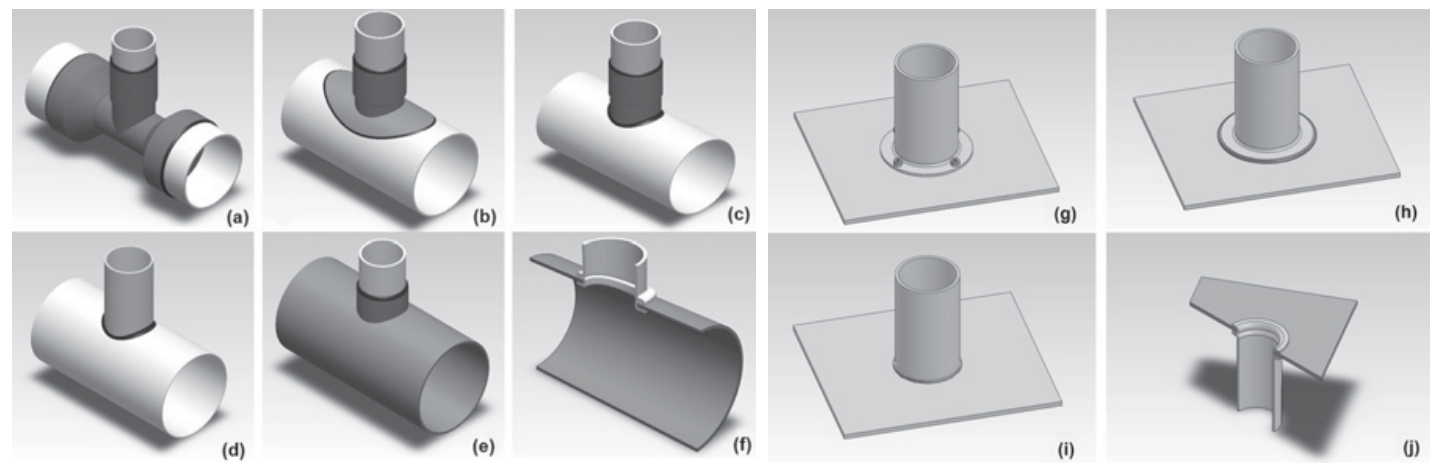

Figure 1. Conventional and new proposed technologies for connecting tubes and fixing tubes to sheets.

Table 1. Summary of the main features of joining by forming and by welding.

\begin{tabular}{|lcc|}
\cline { 2 - 3 } \multicolumn{1}{c|}{} & Joining by forming & Joining by welding \\
\hline Mechanism & Plastic deformation & Melting with addition of filler materials \\
Shape of the connections & Arbitrary geometries & Limited to butt, lap, corner and edge joints \\
Operating temperature & Ambient & Melting point \\
Heat-affected zones & No & Yes \\
Shielding gases & No & Yes \\
Materials & Metals and polymers & Metals (similar) \\
Coated materials & Possible & Very difficult or impossible \\
Energy consumption & Less & More \\
Productivity & More & Less \\
Cost & Less & More \\
Environmental friendliness & More & Less \\
\hline
\end{tabular}

The utilization of mechanical fasteners usually suffers from unwanted aesthetic features, corrosion problems or functional difficulties related to the maximum load that nuts, bolts and rivets can support safely. Welding has similar problems to those mentioned for mechanical fasteners plus specific difficulties. These difficulties arise from dimensional inaccuracies and heat-affected zones resulting from the heat-cooling cycles, from weldability of dissimilar materials (e.g. joining steel or aluminium tubes to aluminium or copper sheets), from the production of undesirable fumes and smokes in fabrication, and from expensive and time consuming issues related to quality inspection of the welds. Structural adhesive bonding offers engineers the possibility of joining different types of materials while improving aesthetics by avoiding rivets and bolt heads. However, adhesives require careful preparation of the surfaces where they are to be applied and may experience significant decrease in performance over time and in the presence of hostile environmental conditions (e.g. prolonged expose in moist environments).

Recent developments in joining by forming that are comprehensively systematized in state-of-the-art reviews by Mori et al. (2013) and Groche et al. (2014) allow concluding that plastic deformation offers great potential to connect tubes and fix tubes to sheets while combining the growing demands for high productivity, low fabrication costs and environmental friendliness with high performance and material versatility. Table 1 summarizes the main differences between joining by forming and by welding.

This paper presents innovative joining technologies that make use of axisymmetric or asymmetric plastic instability waves in thin-walled tubes subjected to axial compression for connecting tubes and fixing tubes to sheet panels at room temperature in situations where the axis of the branch tube or sheet is perpendicular or inclined to the axis of the main body tube (Gonçalves et al. 2014a; Gonçalves et al. 2014b). In case of connecting tubes, the paper will also cover the challenge of joining two tubes by their 

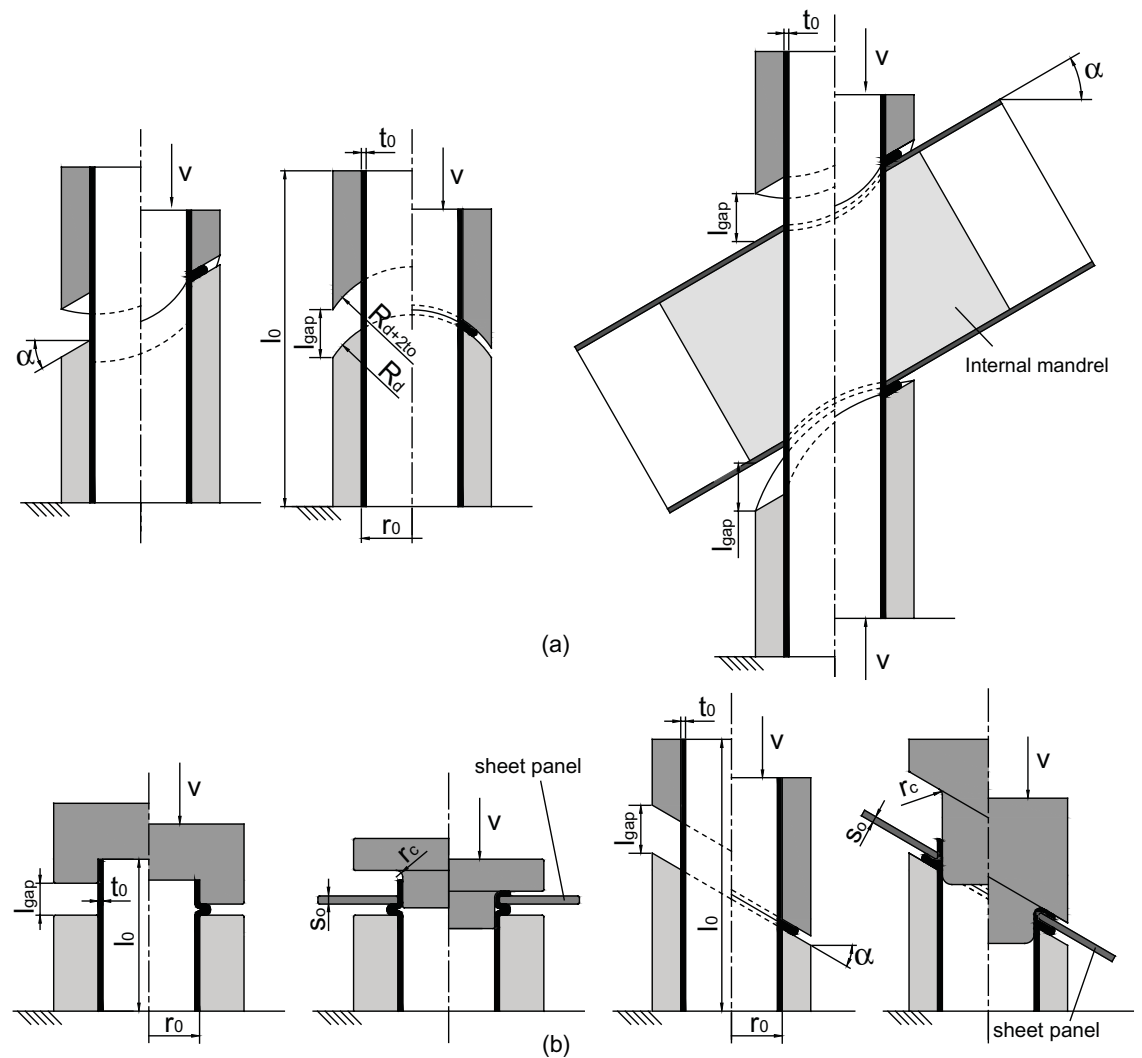

Figure 2. (a) Sectional schematic views of the tool system to develop and propagate inclined, out-of-plane, instability waves in tubes and to produce inclined tube joints. (b) Sectional schematic views of the tool system to develop and propagate inclined, in-plane, instability waves to fix tubes to sheets.

ends. Figures $1 \mathrm{f}$ and $1 \mathrm{j}$ present glimpses of the new proposed technologies in case the axis of the branch tube or sheet is perpendicular to the axis of the main body tube.

Besides allowing connecting dissimilar materials (e.g. metals and polymers) and being successfully employed in fixture conditions that are difficult and costly to achieve by means of conventional joining the new proposed technologies also cope with the growing concerns on the demand, lifecycle and recycling of materials.

\section{New joining technologies}

\subsection{Inclined connections}

The new proposed technologies for connecting tubes and fixing tubes to sheets are schematically shown in Fig. 2. The idea behind the technologies is based on controlling the development and propagation of plastic instability waves in thin-walled tubes subjected to axial compression beyond the bifurcation point in the load-displacement evolution.

In case of tube joining (Fig. 2a) the left and middle schematic drawings show the upper and lower dies that are needed to trigger and propagate inclined, out-of-plane, instability waves between contoured dies at the open and closed positions. The rightmost schematic drawing shows an application of this concept for producing inclined tube joints. The sectional views show the active tool components 

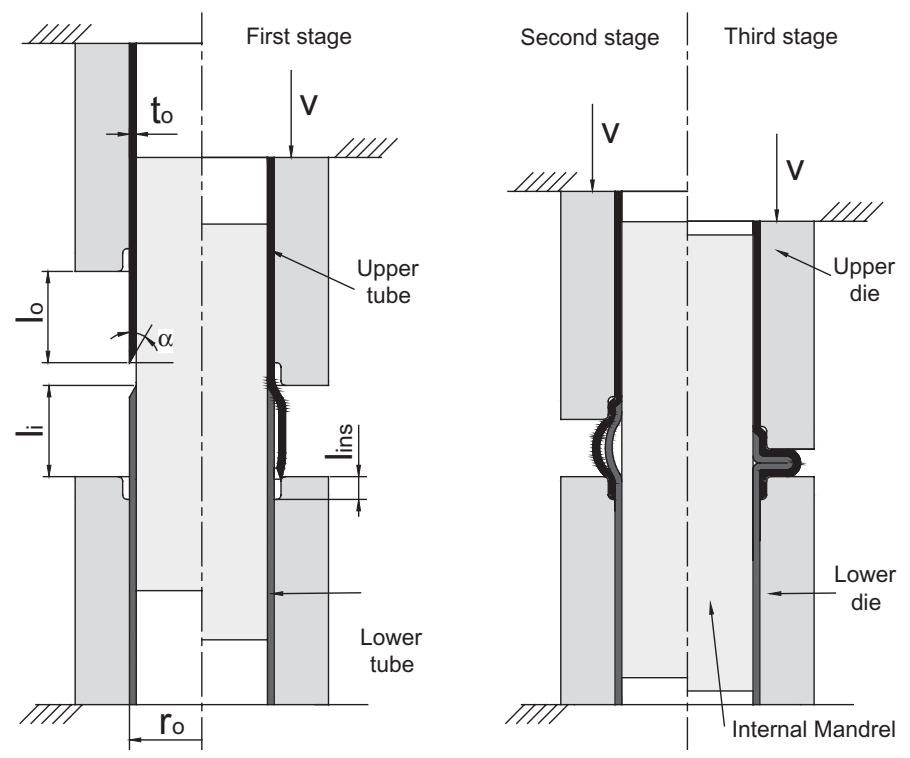

Figure 3. (a) Sectional schematic views of the tool system to connect two tubes by their ends.

consisting of upper and lower contoured dies and internal mandrels (if present). The internal diameter of the dies is dedicated to a specific reference radius $r_{0}$ of the main body tube. The radius $R_{d}$ of the parting out-of-plane surface of the dies together with its inclination $\alpha$ to the axis of the main body tube are dedicated to a specific instability wave or, in case of tube joining, to a specific radius of the branch tube. The difference between the radius $R_{d+2 t_{0}}$ and $R_{d}$ of the upper and lower parting surfaces is crucial to accommodate the plastic compression bead at the end of stroke. The initial gap opening $l_{\text {gap }}$ between the upper and the lower contoured dies controls triggering and propagation of the plastic instability waves namely, the number, width and relative position of the compression beads along the axis of the main tube.

Figure $2 b$ shows two different tool systems utilized for fixing tubes to sheets that make use of axisymmetric (leftmost setup) or asymmetric (rightmost setup), in-plane, plastic instability waves leading the compression beading and axisymmetric or asymmetric tube end flaring. The plastic instability waves are produced by means of appropriate flat or contoured dies whereas flaring is accomplished by compressing the upper tube end with and appropriate radiused punch in order to expand material outwards and form a single-lap inclined flange.

\subsection{End-to-end connections}

The new proposed technology for joining two tubes by their ends is schematically shown in Fig. 3. As seen from the open, intermediate and closed positions of the tool system, joining is accomplished by a sequence of three different cold forming stages that are carried out sequentially in a single stroke: (i) expansion, (ii) local buckling and (iii) clamping by mechanical locking.

Expansion is performed by forcing the upper tube against the chamfered end of the lower tube in order to enlarge the unsupported height $l_{0}$ of the upper tube radially and to create adjacent counterfacing surfaces between the two tubes to be joined. During this stage the chamfered end of the lower tube acts like a tapered punch (refer to "first stage" in Fig. 3).

Once the unsupported height $l_{0}$ of the upper tube reaches the lower edge of the depth of insertion $l_{\text {ins }}$ resulting from the radial clearance between the tube and the upper end of the lower die, there is a 


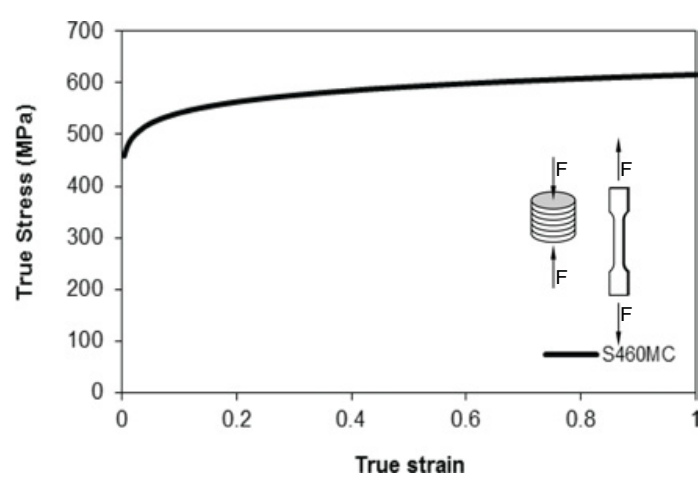

(a)

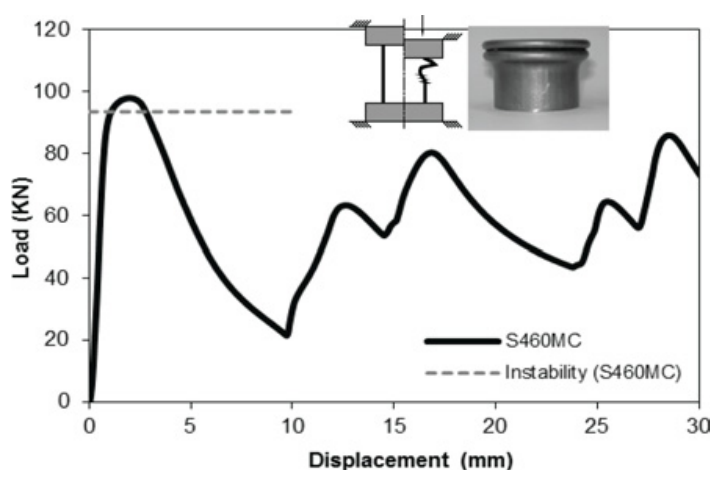

(b)

Figure 4. Mechanical characterization of the commercial S460MC welded tubes. (a) Stress-strain curve and (b) load-displacement curve and critical instability load for the axial compression between flat parallel platens.

sudden change in material flow and plastic instability waves are triggered as a result of local bucking under axial compression loading (refer to "second stage" in Fig. 3). The internal mandrel ensures that the plastic instability waves are formed exclusively outwardly so that design specifications of the inner diameter of the tube joint are met and alterations in the flow of liquids or gases are prevented.

Finally, propagation of the plastic instability waves under continuous axial compression loading clamps the adjacent counterfacing surfaces of the two tubes by mechanical locking (refer to "third stage" in Fig. 3). It is worth noting that although the new proposed joining process has been developed for carbon steel tubes it can also be used in tubes made from other metals or thermoplastics such as polyethylene (PE) and polypropylene (PP).

The main operating parameters influencing the new proposed technologies for inclined and end-toend connections are: (i) the slenderness ratio $l_{\text {gap }} / r_{0}$ between the initial gap opening and the reference radius of the tube, (ii) the ratio $t_{0} / r_{0}$ between the wall thickness and the reference radius of the tube, (iii) the inclination angle $\alpha$ of the contoured dies, (iv) the mandrel (if present) and (v) the tribological conditions. This paper will address the influence of some of these parameters.

\section{Material characterisation}

The experimental developments were performed on commercial S460MC (carbon steel) welded tubes with an outer radius $r_{0}=16 \mathrm{~mm}$ and a wall thickness $t_{0}=1.5 \mathrm{~mm}$ in the "as-received" condition. The stress-strain curve of the tube material was determined by means of tensile and stack compression tests carried out at room temperature on a hydraulic testing machine (Instron SATEC $1200 \mathrm{kN}$ ) with a crosshead speed equal to $100 \mathrm{~mm} / \mathrm{min}$ (Fig. 4a).

The onset of local plastic instability was characterized by compressing tubular specimens with 77.5 and $100 \mathrm{~mm}$ initial length between flat parallel platens. The experimental value of the critical load $P_{c r}$ that is needed for triggering plastic instability waves (or wrinkles) along the tubes was determined experimentally as $P_{c r}=93.5 \mathrm{kN}$ (refer to the dashed horizontal grey line in Fig. $4 \mathrm{~b}$ ).

Further information on the methods and procedures that were utilized to obtain the stress-strain curve and the critical instability load at the onset of plastic instability are available elsewhere (Alves et al. 2012). 


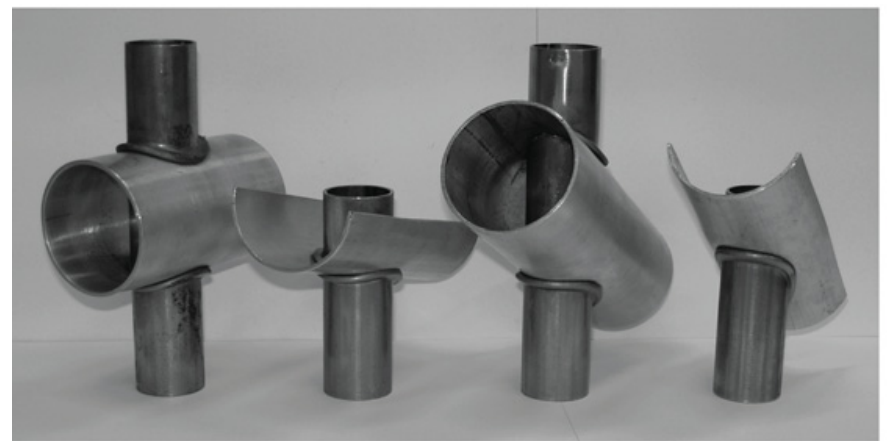

(a)

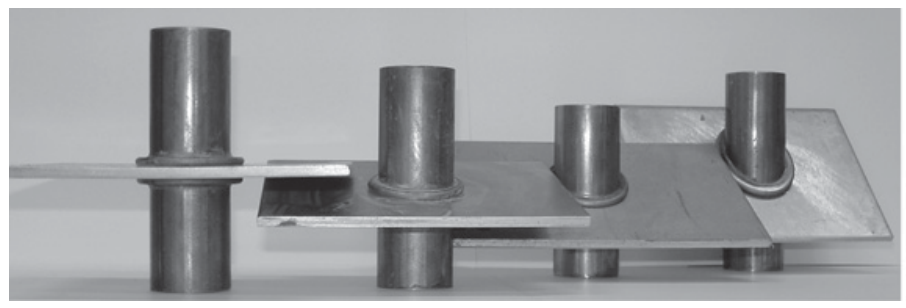

(b)
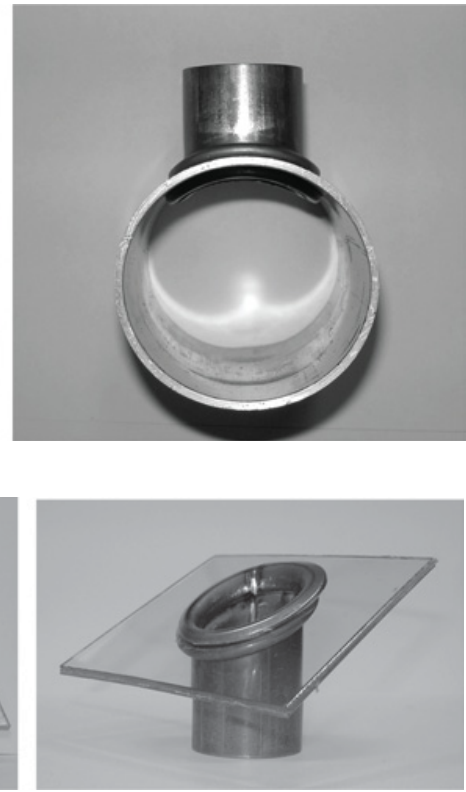

Figure 5. Application of the new proposed technology for (a) connecting full-size and half-sectioned tubes and for (b) fixing tubes to sheets (metallic and polymeric) at different inclination angles.

\section{Results and discussion}

\subsection{Inclined connections}

Figure 5a shows applications of the proposed technology for connecting tubes (or half-tubes) in situations where the axis of the branch tube is perpendicular or inclined to the axis of the main body tube. Figure 5b shows applications for fixing tubes to sheets in situations where the axis of the sheet is perpendicular or inclined to the axis of the main body tube. The solutions can, for example, be successfully employed in the seat bottom frame of automotive as an alternative to welding in order to reduce costs and eliminate heat distortion problems. All the connections shown in Fig. 5 were performed between welded carbon S460MC steel tubes, seamless aluminium AA6062 tubes, aluminium AA5754 sheets and polycarbonate sheets.

Figure 6 shows the results obtained from the numerical modelling of inclined connections that were carried out in the finite element computer program I-Form, which is being developed by the authors since the 1980's (Alves et al., 2004). Figure 6a, for example, shows the initial, intermediate and final predicted geometry of a tube attachment where the axis of the branch tube (labelled as "Tube B") is inclined by $30^{\circ}$ to the axis of the main body tube (labelled as "Tube A"). As seen in the figure, joining is performed by means of two inclined, out-of-plane, plastic instability waves that closely match the intersection of the two tubes. Two different types of mandrels are employed; (i) a conventional internal mandrel placed inside the main body tube ("Mandrel A") and (ii) a special purposed sectioned internal mandrel made of two different parts ("Mandrel B") placed inside the branch tube. The conventional mandrel avoids the development of unacceptable inward plastic flow during triggering and propagation of the plastic instability waves whereas the special purposed sectioned mandrel (allowing for the easy removal of mandrel in practice) prevents the compression beads to plough into the branch tube. The 

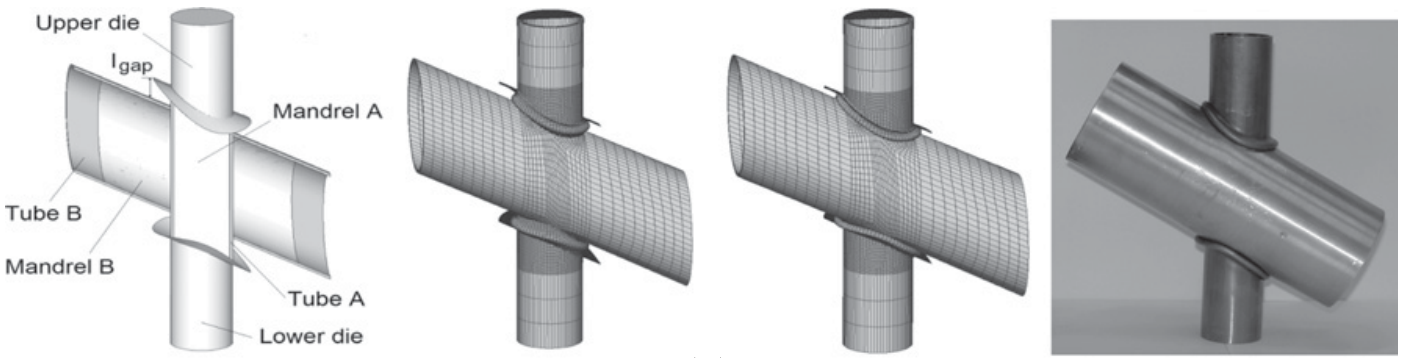

(a)

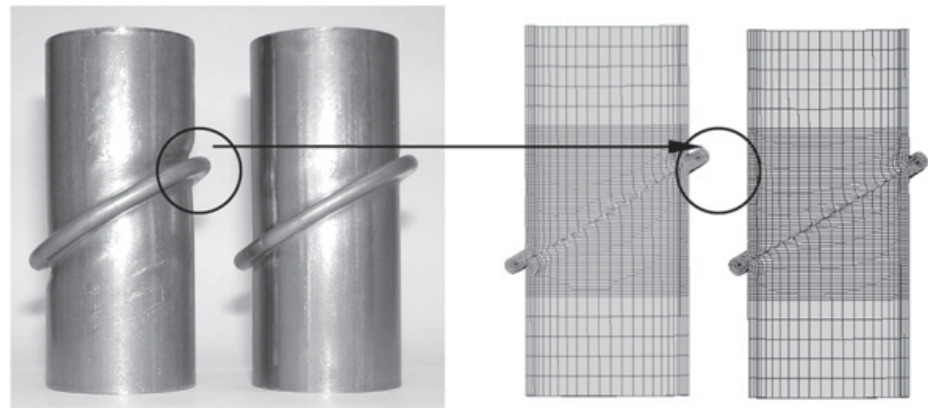

(b)

Figure 6. (a) Finite element model and predicted geometries at the middle and end of stroke with photograph of an inclined connection $\left(\alpha=30^{\circ}\right)$ between two tubes. (b) Photograph and finite element predicted geometries disclosing the influence of the internal mandrel in the development of sound asymmetric, in-plane, plastic instability waves for fixing tubes to sheets.

branch tube behaves as a sleeve during the entire joining process and its internal sectioned mandrel contributes decisively to ensure the overall success of the inclined joining process.

Figure $6 \mathrm{~b}$, allows understanding the role played by the internal mandrel in preventing the development of plastic instability waves exhibiting both inward and outward plastic flow during the fixing of tubes to sheets in situations where the axis of the sheet is perpendicular or inclined to the axis of the main body tube (refer also to Fig. 5b). The compression beads resulting from these plastic instability waves exhibiting both inward and outward plastic flow would lead to non-acceptable joints and, therefore, justify the reason why the utilization of internal mandrels is mandatory to ensure the overall quality and tolerances that are required for the tube-sheet inclined connections.

\subsection{End-to-end connections}

Figure 7 a shows a photograph of several tube specimens that were connected by their ends using the new proposed technology. The observation of the real and finite element predicted cross sections in Figs. 7a and $7 \mathrm{~b}$ allows concluding that the leftmost test sample (corresponding to $l_{\text {gap }} / r_{0}=1.9$ ) does not ensure locking between the two tubes whereas the rightmost test sample (corresponding to $l_{g a p} / r_{0}=4.4$ ) presents a joint with two compressions beads instead of one.

In case of the leftmost test sample, the absence of connection is because the initial unsupported gap height $l_{\text {gap }}$ is not big enough to allow compression beads to develop and lock with each other by plastic instability. In case of the rightmost test sample, the formation of two compression beads instead of one is due to the fact that high values of the initial unsupported gap height $l_{\text {gap }}$ provide conditions for the development of multiple compression beads that will interfere and be placed on top of each other, as they are formed in-between the upper and lower dies. 

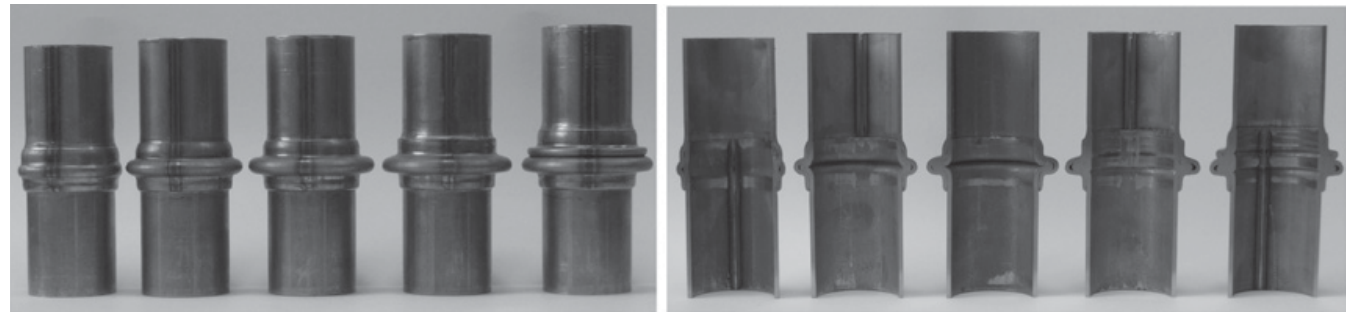

(a)
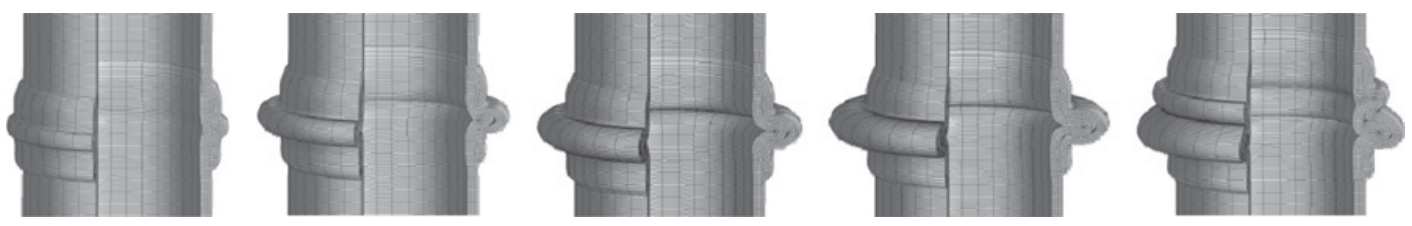

(b)

Figure 7. End-to-end connection of tubes by plastic deformation. (a) Experimental joints and cross sections and (b) finite element predicted geometries for specimens with different values of the slenderness ratio $l_{\text {gap }} / r_{0}$.

Neither the operative conditions corresponding to the leftmost test sample nor those corresponding to the rightmost test sample are acceptable for connecting two tubes by their ends. The process window is therefore restricted to values of the slenderness ratio $l_{\text {gap }} / r_{0}$ in the range between the two abovementioned limits. However, as mentioned by Silva et al. (2015), it is worth noting that the process window must not be confused with the potential range of applicability because the limits on the slenderness ratio $l_{\text {gap }} / r_{0}$ only define the range of values of the unsupported heights of the upper and lower tubes that need to be utilized for successfully connecting any two tubes by their ends.

\section{Conclusions}

The new proposed technologies for connecting tubes and tubes to sheets by triggering and controlling the propagation of plastic instability waves in thin-walled tubes offer several advantages as compared with conventional technologies based on mechanical fixing with fasteners, welding or structural adhesive bonding because they are:

- flexible solutions capable of handling small, medium or large batch sizes with different geometries and high levels of repeatability in production line;

- environmentally friendly solutions that allow savings in raw material and eliminate filler materials and shielding gases;

- energy saving solutions that eliminate heat-cooling cycles as well as heat affected zones in the regions of the tubes and sheet panels that are joined together;

- value added solutions that are capable of connecting tubes and fixing tubes to sheet panels made of dissimilar materials;

- cost-efficient solutions that require low amount of capital investment because they can be designed to operate with existing machine-tools.

Because the new proposed technologies can be successfully employed in fixture conditions that are difficult and costly to achieve by means of conventional technologies they can also foster innovative ideas in product development. 


\section{ICNFT 2015}

The authors would like to acknowledge the support provided by MCG - Mind for Metal and Fundação para a Ciência e a Tecnologia, Portugal under LAETA-UID/EMS/50022/2013.

\section{References}

[1] L.M. Alves, P.A.F. Martins, Journal of Materials Processing Technology 212, 1200 (2012)

[2] L.M. Alves, J.M.C. Rodrigues, P.A.F. Martins, Journal of Engineering Manufacture 218, 1695 (2004)

[3] A. Gonçalves, L.M. Alves, P.A.F. Martins, Journal of Materials Processing Technology 214, 132 (2014)

[4] A. Gonçalves, L.M. Alves, P.A.F. Martins, Steel Research International 85, 67 (2014)

[5] Groche, P., S. Wohletz, M. Brenneis, C. Pabst, and F. Resch. Journal of Materials Processing Technology 214, 1972 (2014)

[6] K.I. Mori, N. Bay, L. Fratini, F. Micari, A.E. Tekkaya, CIRP Annals of Manufacturing Technology 62, 673 (2013)

[7] C.M.A. Silva, C.V. Nielsen, L.M. Alves, P.A.F. Martins, Journal of Cleaner Production 87, 777 (2015) 